\title{
Regulation of the genes involved in neurotransmission in Attention Deficit/Hyperactivity Disorder
}

\author{
Barbara $\mathrm{CuCh}^{1}$, Anna PolaczeK ${ }^{2}$, Magdalena IdziaK ${ }^{3}$, Lidia KotuŁa ${ }^{2}$, \\ Ryszard MaciejewsKi ${ }^{1}$, Barbara Madej-Czerwonka ${ }^{1}$, Janusz Kocki ${ }^{2 *}$ \\ ${ }^{1}$ Chair and Department of Human Anatomy, Medical University of Lublin, Poland \\ ${ }^{2}$ Chair and Department of Human Genetics, Medical University of Lublin, Poland \\ ${ }^{3}$ Chair and Department of Experimental and Clinical Pharmacology, Medical University of Lublin, Poland
}

\begin{tabular}{|c|c|}
\hline ARTICLE INFO & ABSTRACT \\
\hline $\begin{array}{l}\text { Received } 03 \text { June } 2015 \\
\text { Accepted } 15 \text { June } 2015\end{array}$ & Attention Deficit Hyperactivity Disorder is the full name of the disease commonly \\
\hline $\begin{array}{l}\text { Keywords: } \\
\text { genetics, } \\
\text { ADHD, } \\
\text { candidate gene studies, } \\
\text { pharmacogenetics, } \\
\text { methylophenidate, } \\
\text { serotonin, } \\
\text { dopamine, } \\
\text { DRD, } \\
\text { DAT, } \\
\text { 5HT2A. }\end{array}$ & $\begin{array}{l}\text { to } 12 \% \text { of all children world-wide. The current clinical criteria (the base for diagnosis) } \\
\text { can be found in DSM -V. The core symptoms are divided in three groups: hyperactivity, } \\
\text { impulsivity and impaired attention. The aetiology of the disorder is combined, including } \\
\text { a wide range of factors, and the genetic, environmental, toxic, perinatal background is } \\
\text { taken into account. Because, currently, more and more studies are seeking to explore the } \\
\text { heritability of the disorder, the aim of this study is to review the information provided by } \\
\text { different research centres which discuss the genetic background of the disease. Herein, } \\
\text { we present the results of different studies gathered from the online database. Our findings } \\
\text { indicate that the participation of genetic factors within this disorder is supported by } \\
\text { family, twin and adoption studies. Indeed, in current literature, researchers estimate that } \\
\text { there is a higher risk of developing ADHD among children from families with an ADHD } \\
\text { history. Of particular note is that there are some studies indicating particular genes that } \\
\text { determine the susceptibility to ADHD. Such studies make mention that most of these } \\
\text { genes encode components of the dompaminergic and serotoninergic neurotransmission } \\
\text { systems. Researchers in the field, thus, are attempting to link the presence of certain } \\
\text { alleles in affected children with their response to treatment. Yet, while ADHD is now } \\
\text { considered as being a disorder of genetic background, we cannot indicate a single gene } \\
\text { or its mutation that would be crucial in the aetiology and diagnosis. Still, a number of } \\
\text { candidate genes have been reported so far. }\end{array}$ \\
\hline
\end{tabular}

\section{INTRODUCTION}

Attention Deficit/Hyperactivity Disorder is a frequent mental disorder among children. Indeed, it is estimated that over $5 \%$ of all young people worldwide present symptoms of ADHD [9]. The disease is characterized by increased inattentiveness, hyperactivity and impulsivity. Such is the nature of the disease that we can be even unconscious of the presence of children with ADHD in our surroundings. Yet, it is assessed that the symptoms of ADHD persist into adulthood in 30\% of all patients [14]. On the other hand, there are children misdiagnosed with ADHD. They carry a stigma without any reason - just because of the lack of clear criteria for diagnosis. This is what causes misunderstanding

\footnotetext{
* Corresponding author

e-mail: janusz.kocki@tlen.pl
}

of this disorder. It seems important to analyse this issue as both a health and social subject [9]. In our work, we aimed to find accurate information about the symptoms, background and treatment possibilities with regard to ADHD. Of note, we concentrate attention towards the genetic approach to this problem as being most promising for its understanding.

Methods of ADHD diagnosis are still being improved. In May 2013, new diagnostic criteria for ADHD was published in the fifth edition of the Diagnostic and Statistical Manual of Mental Disorders. Therein, the American Psychiatric Association introduce some changes compared to DSM-IV and ICD-10 held criteria, which were considered essential before. This previous criteria were based on the behavioural symptoms presented by affected children, and they demanded a prevalence of symptoms in more 
than one context, as well as the exclusion of other behavioural, mental and development disorders. According to the established scoring system, symptoms had to be chronic (present for 6 months), functional impairing and observed in children before age 7 years. The DSM- IV criteria also broke down ADHD features into two groups: inattentive and hyperactive-impulsive. This approach enabled placement of patients into three categories: a 'combined type', wherein, the afflicted met at least six of the nine symptoms of inattention and six of the nine symptoms of hyperactivity and impulsivity; inattentive (ADHD-I) and hyperactive (ADHD-H) types, wherein the assessed demonstrated the dominance of one kind of symptom. The ICD-10 scale contained more restricted criteria for diagnosis. It linked the features of inattention, hyperactivity and impulsivity inside the one term ADHD, and there was no partial diagnosis available [15]. Despite this restriction, there was still numerous difficulties in diagnosis.

In the current criteria, the American Psychiatric Association has tried to eliminate these by introducing some changes. The age criterion was raised to requiring the presence of symptoms by age 12 years or earlier. Moreover, the DSM-V criteria accepts the coexistence of ADHD with autistic spectrum disorders (which had to be excluded according to DSM-IV). The same symptoms as divided into two main groupings, are used in the new scale. However, new type-examples was added to suit the assigned criteria to the age of the individual. What is more, the criteria for adults has changed. In DSM-V, the set of symptoms which were required for diagnosis as an adult were reduced to being the presence of five from each domain: inattention and impulsivity/hyperactivity. The new criteria for ADHD is presented in Table $1[6,7]$.
The new manual indicates that brain developmental impairments are implicated in ADHD, as it places this entity within the chapter: Neurodevelopmental Disorders. However, according to some research, DSM-V has some omissions that must be addressed through continuous updating and through generating more precise diagnosis for this entity.

By the last half of the Twentieth Century, it was thought that ADHD comes about by damage to the CNS by way of pre- and/or perinatal disorders and by impaired emotional development of the child [11]. However, more recent clinical observations indicate a genetic predisposition. Recent work suggests that about 20 genes involved in neurotransmission are susceptibility genes in ADHD. These genes regulate the transmission of stimuli, and they encode the neurotransmitters and their receptors. In our work, we have noted ADHD research pertaining to dopamine, serotonin, acetylcholine, norepinephrine and GABA receptor genes as being involved in the aetiology of ADHD [8,9]. Hence, neurotransmission disorders are implied as being responsible for ADHD behaviour. Thus, this means that ADHD symptoms arise from biochemical and physiological dysfunctions of the brain. Knowledge of the biological background of the disease, we feel, is crucial in understanding and treating the disease. In this work, certain structures of the human brain have been well examined vis a vis their role in ADHD. However, the theory that disturbances in neural signalling contributes to this disorder is still being assessed. Regarding ADHD, the pre-frontal cortex, striatum, thalamus, hypothalamus and nuclei basal clearly seem to be involved. These regions are known as being responsible for behaviour control, concentration, mood, as well as for mental and physical tiredness. Impaired activity of these regions can result in symptoms which are specific for ADHD. Among these are

\section{Table 1. DSM-V criteria for ADHD[5]}

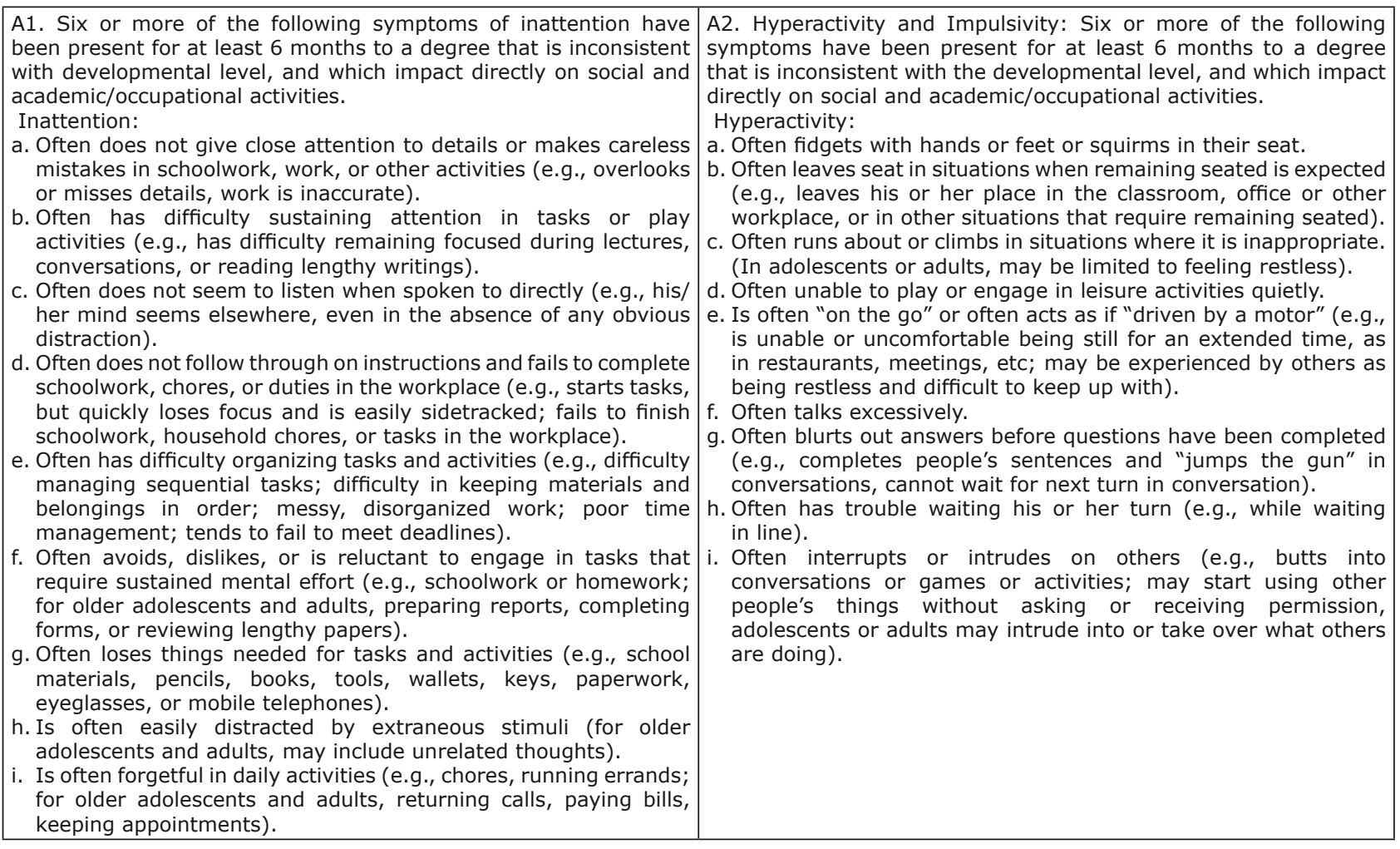


hyperactivity, impulsivity, susceptibility to be distracted, insomnia or low request for sleep, talkativeness and motor anxiety. The effects of the neurotransmitters (serotonin, norepinephrine dopamine and GABA) involved in the neural connections found in these areas, are regulated mainly by the function of their receptors and by degrading enzymes. These control the amount of neurotransmitter within the synapse.

In the case of serotonin, there are three kinds of receptors indicated. The first group consists of receptors 5HT1A and $5 \mathrm{HT} 1 \mathrm{~B}$, both of which bring about a decrease in the amount of serotonin within the synapse. These auto-receptors come into play so as to stop serotonin secretion if there is a lot of this neurotransmitter within the synapse. The second group of serotonin receptors, the post-synaptic, are that of 5HT2A, 5HT3, 5HT4, as well as several others. These are situated inside the next cell of the neural chain, and these act by transduction of signals. They are commonly allied to the effects of protein $\mathrm{G}$ in this cell. The last group of receptors for the serotonin neurotransmitter are that of the serotonin transporter (SERT). This is responsible for reuptake of serotonin from the synapse, for transport to within the pre-synaptic neuron, where it is stored in synaptic bubbles. SERT also decreases the amount of serotonin inside the synapse.

Regarding norepinephrine, the manner of regulation is similar to that of serotonin. Indeed, it is served by the same kind of receptors to give the same effects. As to dopamine, which is of documented significance vis a vis mental disorders, five post-synaptic receptors are indicated - D1, D2, D3, D4 and D5. The function of dopamine is also regulated by reuptake by the pre-synaptic dopamine transporter (DAT). Both dopamine and norepinephrine are removed from the synapse by the enzymes: COMT (catechol-O-methyltransferase) and MAO (monoamine oxidase), which also break up serotonin.

GABA (gamma-aminobutyric acid) is known as being one of the main inhibiting transmitters. It is controlled by way of the pre-synaptic GABA transporter (GAT) and by certain post-synaptic receptors. Recent work has brought about the knowledge that some of the GABA post-synaptic receptors engineer their effect via ion channels [16].

Knowledge of brain functioning is essential in the understanding of ADHD. Apart from the aforementioned biochemical studies, imaging trials have been undertaken in an attempt to further knowledge of ADHD. Researchers in this field commonly employ fMRI (functional magnetic resonance imaging) to examine which brain regions are activated in children with ADHD. In such studies, these children-subjects are given certain tasks to complete. When they perform these tasks, fMRI creates a map of their brain, hence, denotes the activated regions. The results of such work has revealed that some neural pathways are disrupted within those afflicted with ADHD. Moreover, they partially employ alternate brain pathways compared to healthy children. What is more, according to some studies, differences to the unaffected appear in the frontal, parietal and occipital lobes, the basal ganglia and cerebellum. One such study also suggests that ADHD children exhibit decreased activity in the frontostriatal regions [3]. It should be noted, however, that despite the growing consciousness of the biochemical and neural background of ADHD, the results of such work is conflicted and not yet exploitable for remedial treatment.

Besides the specific brain neurotransmission mechanics seen within the ADHD child, the genetic background of this abnormality is still not known. In studying ADHD, paediatric researchers commonly analyse the genes understood to be involved in important brain signalling pathways. Such work has been done by way of family and twin studies. In 1970, it has been estimated that $20 \%$ of ADHD subjects have affected parents. Furthermore, retrospective researches have particularly revealed symptoms of ADHD in the forefathers of children diagnosed with ADHD. Numerous twin studies have also reported a higher monozygotic to dizygotic twin ratio. What is more, some studies have uncovered a tendency toward antisocial behaviour, mental disorders and alcoholism among the fathers of ADHD children. This all seems to indicate the existence of a form of persistent inherited ADHD.

At the end of the Eighties of the Twentieth Century, Goodman and Stevenson estimated that in $64 \%$ of all cases, the symptoms of ADHD are inherited. Later, Edelbrock suggested that problems with sustained attention are more frequent among monozygotic twins (68\%), compared to dizygotic ones (29\%). The same ratio was observed in regard to reading disabilities in this examined group. Towards the end of the Twentieth Century, a family study was performed involving twins and their siblings. The study subjects were examined by way of utilizing the then current DSM III-R and DSM IV scale. This study estimated that ADHD heritability achieve a level of even $91 \%$ [11]. The heritability of ADHD and co-morbidities as put forward by different researchers is presented in Table 2.

Table 2. Heritability of ADHD co-morbidities according to different researchers [14]

\begin{tabular}{|l|c|c|c|}
\hline \multicolumn{1}{|c|}{ Disorder } & ADHD & $\begin{array}{c}\text { Attentional } \\
\text { problems }\end{array}$ & $\begin{array}{c}\text { Reading } \\
\text { disabilities }\end{array}$ \\
\hline Monozygotic & $81 \%$ & $68 \%$ & $84 \%$ \\
\hline Dizygotic & $29 \%$ & $29 \%$ & $64 \%$ \\
\hline Author & Gilger et al. & Gilger et al. & Edelbrock et al. \\
\hline
\end{tabular}

Recent advances in molecular genetics have allowed the use of modern methods for examining families affected by ADHD. Indeed, genotyping has enabled the indication of ADHD susceptibility genes. The genome-wide association study (GWAS), which was first employed at the end of the Twentieth Century, is a kind of hypothesis-free analysis of the genome, and is felt to be useful in in identifying the risk alleles within polygenic disorders like ADHD. The transmission disequilibrium test (TDT) is the second important tool that has been developed and utilize in ADHD studies. TDT enables a comparison of the transmission of candidate alleles among families [2].

In such work, the most frequently investigated genes are these belonging to the dopaminergic neurotransmission system: DAT1, DRD4, DRD5. Herein, we will try to characterize these genes and their probable role in ADHD aetiology.

The first, the DAT1 (SLC6A3 gene) is located on chromosome $15(15 \mathrm{p} 15.3)$. It encodes a dopamine transporter and is expressed primarily in both the striatum and the substantia 
nigra. An important feature seen in ADHD patients is a 40bp variable number tandem repeat (VNTR) polymorphism in the 3'-untranslated region of DAT1. The 480bp allele of this gene (10-repeat allele) is considered as being associated with ADHD (OR 1.13). TDT work has, however, failed to indicate sufficient transmission of this allele. Studies analysing genes and their correlations with the expression of particular symptoms, estimate that a 10-repeat allele is associated with high reaction time variability. Loo and colleagues, put forward that people showing a gene structure that is homozygous with $480 \mathrm{bp}$ are more impulsive and hyperactive, compared to individuals displaying the same homozygous with other alleles. Yet, in follow-up work, no differences between being homozygous and heterozygous in terms of outward effect were found. As mentioned, the dopamine transporter acts by enabling dopamine re-uptake from the synapses. This function is most significant in the striatum. Moreover, a lower expression of gene is observed in the prefrontal cortex of those afflicted with ADHD. In this part of the brain, the dopaminergic system function depends on the synthesis and the metabolism of dopamine, rather than on its re-uptake. In the substantia nigra, DAT1 is thought to play a role in the regulation of dopamine secretion as well, although, this situation would be specific for this region of the brain alone $[8,13]$.

DRD4 - the second susceptibility gene of consequence, is located on chromosome 11 (11p15.5). It encodes a dopamine receptor and is noted to be of the highest density in the prefrontal cortex-region of the brain. This brain structure is responsible for attention, cognitive function and behaviour control. Polymorphism of the exon 3 (2-7 copies of a $48 \mathrm{bp}$ repeat sequence) is evident in those displaying ADHD [13]. This exon encodes the part of the protein crossing the cell membrane of the neuron. Its function is crucial for enabling the dopaminergic system inside the prefrontal cortex and the striatum. Researchers suggest that the presence of a 7-repeat allele evidences an enhanced risk of ADHD (OR 1.34). Moreover, there is a significant preferential transmission of this condition, from parents to children with ADHD (as assessed by way of TDT). The presence of a 7-repeat allele results in an decreased response to DA, especially inside the prefrontal cortex. This allele also appears to encode for less efficient receptors in adenylate cyclase activation (a key regulatory enzyme). Additionally, the 7-repeat allele seems to bring about less gene expression. According to some studies, children carrying this variant show longer response times (Swanson and colleagues) and greater impulsiveness (Langley and colleagues).

$D R D 5$ is located on chromosome 4. It encodes the dopamine receptor which activates adenyl cyclese, and is expressed particularly in the limbic area. Regarding ADHD, the polymorphism noted in ADHD studies exists at the beginning of this gene's structure. Therein, the dinucleotide block (CA) is seen to be repeated multiple times. The size of this segment ranges from 134 to 156 base pairs, and the variant that is 148-base pairs long is associated with an increased risk of ADHD (OR 1.24-1.57), particularly with regard to impaired attention. Researchers, by way of TDT work, have noticed a trend towards transmission of this allele and an association with the persistence type of ADHD.
Additional ADHD studies see that alterations in serotonin neurotransmission are also involved in in this disorder. Indeed, some of such studies have uncovered altered concentrations of 5-hydroxyindoleacetic acid in the cerebrospinal fluid of patients with ADHD. Follow-up studies of the pharmacological effects of drugs regulating serotonine function additionally reinforce the significance of its role in ADHD aetiology. What is more, reduction of ADHD symptoms is frequently achieved with serotonin agonists or serotonin re-uptake inhibitors. For instance, fluoxetine administration has been seen to induce reduction of hyperactivity in experiments involving DAT1-KO mice. Such work provides evidence that various neuronal pathways are involved in ADHD, besides that of dopaminergic [8]. However, some studies of this issue estimate that response to treatment depends on patient's genotype.

So far, genetic variation in serotoniergic signalling has been noted in a few genes. These being: DDC, MAOB and 5HT2A. Their significance is presented in Table $3[2,8]$.

Table 3. The etiology of ADHD and serotoninergic gene variants

\begin{tabular}{|c|c|c|c|}
\hline Gene & DDC & MAOB & $5 \mathrm{HT} 2 \mathrm{~A}$ \\
\hline Function & $\begin{array}{c}\text { Essential role in } \\
\text { synthesis }\end{array}$ & $\begin{array}{c}\text { Involved in } \\
\text { degradation }\end{array}$ & $\begin{array}{c}\text { Encode serotonin } \\
\text { receptor }\end{array}$ \\
\hline Allele & $\begin{array}{c}\text { Four-marker } \\
\text { haplotype - CAAT }\end{array}$ & $\begin{array}{c}\text { Three-marker } \\
\text { haplotype - CGC }\end{array}$ & $\begin{array}{c}\text { Three-marker } \\
\text { haplotype - GCC }\end{array}$ \\
\hline OR & $\begin{array}{c}\text { In combined ADHD } \\
\text { (both adults and } \\
\text { children) }\end{array}$ & In adult ADHD & $\begin{array}{c}\text { In combined ADHD } \\
\text { (both adults } \\
\text { and children) } \\
\text { SNP T102C }\end{array}$ \\
\hline \multicolumn{2}{|c|}{2.04} & 1.9 & 1.55 \\
\hline
\end{tabular}

Additional research in ADHD, indicate that other susceptibility genes are involved in the disorder's etiology. One of these, TPH2, encodes for tryptophan hydroxylase. A haplotype block associated with ADHD is seen as the result of a construction of multiple SNPs within this gene [1]. COMT has also come under consideration. The genetic instructions for making catechol-O-methyltransferase is contained in COMT. This enzyme is involved in the metabolism of several brain neurotransmitters. Regarding COMT, previous studies have brought about a focus upon a Val/Met polymorphism in codon 158 . When this is homozygous for methionine production, this appears to bring about a 3to 4-fold reduction of COMT activity. This has significant effect with regard to brain signalling pathways. It must be noted, however, divergent findings in the case of Val158Met polymorphism are available $[8,10]$.

$C L O C K$ is also considered as being a susceptibility gene for ADHD symptomology, especially in connection with patients displaying day activity destruction and sleep request impairment. Its sequence encodes a transcription factor essential for the regulation of many circadian output genes. Indeed, some studies suggest that the expression of up to $10 \%$ of all human genes can be dependent on CLOCK. One of its alleles (rs 1801260 with T) is observed to be more frequent among ADHD patients with delayed sleep phase syndrome (DSPS) [18].

Besides the previously stated areas of ADHD study, pharmacogenomic research is also actively pursued. In such work, researchers endeavour to ascertain the extent of a relationship between genotype and response to the treatment, as well as to understand the biochemical background of such 
connections. By way of such work, the first-line treatment for severe ADHD include stimulants like methylphenidate $(\mathrm{MPH})$ and dexamfetamine, as well as non-stimulants such atomoxetine.

$\mathrm{MPH}$ is most commonly used for alleviating the signs and symptoms of ADHD (having been prescribed for over fifty years), and is considered highly efficient. However, its way of action is still unclear. What we do know nowadays, is that methylophenidate induces an increase of dopamine and norephinephrine inside the extra-neuronal space. This probably comes about due to the effect of DAT blocking and to dopamine re-uptake inhibition [17]. Research has revealed that an increased concentration of dopamine improves receptor stimulation and signal transduction. What is more, the amplitude of post-synaptic neurons decreases because less dopamine is stored in the pre-synaptic neurons bubbles because of dopamine transporter blockage. This mechanism is especially important when patients have difficulties with motor inhibition. The highest brain concentration of methylophenidate appears 60-90 minutes after administration of MPH, while the therapeutic effect lasts about 6-8 hours. What is more, there is no developed tolerance to this medication that has been seen in in vivo tests [13].

As to the serotoninergic system, effective treatment has been demonstrated by the observed effects of MPH administration on serotonin agonists and serotonin reuptake inhibitors, however, some studies of this issue estimate that response to treatment depends on patient's genotype. In such work, researchers have shown that if homozygous with a 10-repeat $D A T$ allele, individuals have worse response to MPH (12 to 14 patients without treatment effect) in comparison to being heterozygous (5 to 16 without treatment effect). In addition, subjects without this 10-repeat $D A T$ allele display the best improvement due to MPH administration, and are most susceptible to treatment. Of note: homo-zygotes have increased expression of $D A T$ in their basal ganglia, and this seems to be the cause of methylophenidate-resistance [12].

There are no doubts of ADHD having a genetic background, although we should remember that the connection between displaying ADHD and the presence of some of the alleles mentioned previously, is not decisive. Gene expression is also an important issue. For instance, environmental factors have influence on child in uteri, as well as after birth. Known risk factors for ADHD include biological and psychosocial conditions, such as family dysfunction brought about by addictions or conflicts. Moreover, pregnancy abnormalities, neonatal asphyxia, prematurity, short time of breastfeeding, deficiency of vitamins and preservatives in food are also under consideration as risk factors.

In conclusion, we should emphasize that there is no single gene evidenced in the etiology of ADHD, as numerous susceptibility genes have been indicated. These can bring about ADHD etiology by their sole presence, as well as by different correlations within the patient's genome. Such variances in effect can be also an explanation to the different phenotypes expressed by the disease. What can be said in summary, is that achievements in genetic research and the resulting treatment possibilities of ADHD studies are promising for patients. Although, more work in this areas are necessary.

\section{REFERENCES:}

1. Baehne, C.G. et al.: Tph2 gene variants modulate response control processes in adult ADHD patients and healthy individuals. Mol. Psychiatry, 14, 1032-1039, 2009.

2. Banaschewski, T. et al.: Molecular genetics of attention-deficit/ hyperactivity disorder: an overview. Eur. Child Adolesc. Psychiatry, 19, 237-257, 2010.

3. Dey, S., Rao, A.R. and Shah, M.: Exploiting the brain's network structure in identifying ADHD subjects. Frontiers in system neuroscience, 6 (2012), http://journal.frontiersin.org/article/10.3389/ fnsys.2012.00075/abstract.

4. DeYoung C.G. et al.: Tremblay, R.E., Kennedy, J.L. and Palmour, R.M. The Dopamine D4 Receptor Gene and Moderation of the Association Between Externalizing Behavior and IQ. Arch. Gen. Psychiatry, 63, 1410-1416, 2006.

5. http://www.cdc.gov/ncbddd/adhd/diagnosis.html

6. http://www.drthomasebrown.com/dsm-5-changes-in-adhddiagnostic-criteria/

7. http://www.pro.psychcentral.com/2013/dsm-5-changes-attentiondeficit-hyperactivity-disorder-adhd/004321.html

8. Kebir O. et al.: Candidate genes and neuropsychological phenotypes in children with ADHD: review of association studies. J. Psychiatry Neurosci., 34, 88-101.1, 2009.

9. Kustanovich V. et al.: Transmission disequilibrium testing of dopamine-related candidate gene polymorphisms in ADHD: confirmation of association of ADHD with DRD4 and DRD5. Mol. Psychiatry, 9, 711-717, 2004.

10. Levy F.: What do dopamine transporter and catechol-omethyltransferase tell us about attention deficit hyperactivity disorder? Pharmacogenomic implications. Aust. N. Z. J. Psychiatry, 41, 10-16, 2007.

11. Levy F., Barr C., Sunohara G.: Directions of etiologic research on attention deficit hyperactivity disorder. Aust. N. Z. J. Psychiatry, 32, 97-103, 1998.

12. Lowe N., Barry E., Gill M., Hawi, Z.: An Overview of the Pharmacogenetics and Molecular, Genetics of ADHD. Current Pharmacogenomics, 4, 231-243, 2006.

13. Madras B. K., Miller G.M., Fischman A.J.: The Dopamine Transporter and Attention-Deficit/Hyperactivity Disorder. Biol. Psychiatry. 57, 1397-1409, 2005.

14. Ribases M.et al.: Exploration of 19 serotoninergic candidate genes in adults and children with attention-deficit/hyperactivity disorder identifies association for 5HT2A, DDC and MAOB. Mol. Psychiatry, 14, 71-85, 2009.

15. Steinhausen H.C.: The heterogeneity of causes and courses of attention-deficit/hyperactivity disorder, Acta Psychiatr. Scand., 120, 392-399, 2009.

16. Stahl S.M.: Leki stabilizujące nastrój, Asteria, Gdańsk, 17, 25-40, 2010.

17. Takon I.: Clinical use of a modified release methylphenidate in the treatment of childhood attention deficit hyperactivity disorder. Annals of General Psychiatry 10 (2011), http://www.annals-generalpsychiatry.com/content/10/1/25

18. Xu X. et al.: Association study between a polymorphism at the 3'-untranslated region of CLOCK gene and attention deficit hyperactivity disorder. Behavioral and Brain Functions, 6 (2010), [http://www.behavioralandbrainfunctions.com/content/6/1/48]. 